\title{
ANSPRUCH AUF ENTWICKLUNGSHILFE?
}

\author{
Dr. Dr. Albert Bleckmann
}

Der Aufsatz von Hermann Weber über den ,,Anspruch auf Entwicklungshilfe“ gibt Veranlassung zu einer kurzen Erwiderung, weil Weber die Probleme nur anreißt und durchaus nicht vollständig ist. Neben dem von Weber geprüften Problem, ob ein Anspruch auf Entwicklungshilfe aus dem Gedanken der Wiedergutmachung und des Sozialstaatsprinzips fließt, treten nämlich die Fragen, ob in der ständigen Praxis der Gewährung von Entwicklungshilfe Völkergewohnheitsrecht begründet ist, ob die Charter of Economic Rights and Duties of States von 1974 Ansprüche auf Entwicklungshilfe begründet, ob solche Ansprüche ferner aus den internationalen Menschenrechtspakten abgeleitet werden können. Diese Fragen sollen hier kurz erörtert werden:

1. Mir scheint zunächst, daß die ständige Praxis der entwickelten Staaten, Entwicklungshilfe zu gewähren, sowohl unter dem Aspekt der Bildung von Völkergewohnheitsrecht als auch aus dem Vertrauensgrundsatz und dem völkerrechtlichen Diskriminierungsverbot heraus rechtlich nicht völlig unbeachtlich ist:

a) Bei der Frage, ob aus der ständigen Gewährung von Entwicklungshilfe durch die reichen Staaten eine Pflicht des Völkergewohnheitsrechts fließt, handelt es sich nicht um das bekannte Problem, ob und wann sich aus völkerrechtlichen Verträgen Völkergewohnheitsrecht entwickeln kann'1. Hier soll nämlich nicht der Inhalt der Verträge in Völkergewohnheitsrecht erwachsen, sondern die vorausliegende Pflicht zum Abschluß solcher Verträge. Nun wird man sicherlich die notwendige Rechtsüberzeugung der Staaten von einer solchen rechtlichen Pflicht nicht aus ausdrücklichen Äußerungen der entwickelten Staaten ableiten können. Die reichen Staaten waren insofern stets sehr vorsichtig und haben sich auch vor der Verpflichtung gescheut, einen bestimmten Prozentsatz ihres Bruttosozialprodukts der Entwicklungshilfe zu widmen. Immerhin wird man aber davon ausgehen dürfen, daß die reichen Staaten heute zumindest eine moralische Pflicht zur Hilfe bejahen. Uberdies braucht die Rechtsüberzeugung der Staaten nicht gerade bei der Praxis, der Gewährung der Entwicklungshilfe, geäußert worden zu sein. Sie kann insbesondere aus der Gesamtheit der im folgenden zu entwickelnden Gesichtspunkte für einen Anspruch auf Entwicklungshilfe fließen. Die Tatsache, daß die entwickelten Staaten stets Entwicklungshilfe geleistet haben, würde dann die anderen Anspruchsgrundlagen verstärken können.

b) Der Vertrauensschutzgrundsatz ist Grundlage zahlreicher Rechtsinstitute des Völkerrechts $^{2}$. Aus diesen einzelnen Rechtsinstituten des Völkerrechts und analogen Rechtsinstituten des nationalen Rechts fast aller Staaten ${ }^{3}$ kann ein allgemeiner Rechtsgrundsatz des Völkerrechts abgeleitet werden, der neue, völkergewohnheitsrechtlich noch nicht verankerte Rechtsinstitute des Völkerrechts tragen kann. Diese „Rechtsanalogie“4 hat sich auch die Wiener Vertragsrechtskonferenz zunutze gemacht, als sie in Art. 69 II wegen des Vertrauens der Vertragspartner Rechtsfolgen eines an sich ungültigen Vertrages aufrechterhielt, die nach Völkergewohnheitsrecht an sich nicht begründet sind. Dieses Vertrauensschutzprinzip kann den Anspruch des Entwicklungsstaates A gegen den reichen Staat B tragen, wenn der Staat B ein bestimmtes Projekt längere Zeit gefördert hat und durch den Abbruch dieser Förderung

\footnotetext{
* VRU 11 (1978), S. 5 ff.

1 Dazu neuerdings K. Doehring, Gewohnheitsrecht aus Verträgen, ZaöRV 36 (1976), S. 77.

2 Vgl. J. P. Müller, Vertrauensschutz im Völkerrecht, 1971.

3 Vgl. etwa C.-W. Canaris, Die Vertrauenshaftung im deutschen Privatrecht, 1971.

4 Vgl. A. Bleckmann, Analogie im Völkerrecht, AVR 1977, S. 161.
} 
die Eigenaufwendungen des Staates A sinnlos werden ${ }^{5}$. Auch die Zusage von Entwicklungshilfe wird man neben der Verbindlichlichkeit einseitiger Versprechen ${ }^{6}$ auf den Vertrauensschutzgrundsatz stützen können.

c) Ein allgemeines Diskriminierungsverbot entsprechend unserem Art. 3 GG ist dem Völkerrecht zwar fremd ${ }^{7}$. Wenn aber die Bundesrepublik zahlreiche Entwicklungsstaaten in der gleichen Situation wie der Bewerber X ständig gefördert hat und die Haushaltsmittel der Bundesrepublik nicht erschöpft sind, wird man angesichts der Dichte des Vertragsnetzes der Bundesrepublik die Ablehnung der Förderung des X als eine verbotene Diskriminierung ansehen müssen ${ }^{8}$.

2. Die Charter of Economic Rights and Duties of States von $1974^{9}$ begründet ihrem Wortlaut nach ${ }^{10}$ in zahlreichen Bestimmungen Pflichten der entwickelten Staaten zur Hilfe in den verschiedensten Formen an die Entwicklungsstaaten. Umstritten ist insofern aber, ob die an sich nach der Satzung der UNO nicht bindende Resolution der UN-Vollversammlung als authentische Interpretation der Satzung ${ }^{11}$, als Völkergewohnheitsrecht ${ }^{12}$, als allgemeiner Rechtsgrundsatz des Völkerrechts ${ }^{13}$ oder als neue, neben die Rechtsquelle des Art. 38 IGH-Statut tretende Rechtsquelle ${ }^{\mathbf{1 4}}$ bindet. Für diese Fragen kann hier auf den ausführlichen Aufsatz von Tomuschat ${ }^{15}$ hingewiesen werden. Aber selbst Tomuschat und Frowein ${ }^{16}$ gelangen zu der Uberzeugung, daß trotz des Widerspruchs der westlichen Industriestaaten die Rechtsäußerung von 120 Staaten der östlichen und der Dritten Welt nicht völlig unverbindlich ist, sondern zumindest die noch mögliche rechtliche Argumentation einschränkt. Diese Diskussion soll hier nicht wieder aufgegriffen werden. Es soll hier vielmehr nur auf einige neue Möglichkeiten hingewiesen werden, den Resolutionen der UN-Vollversammlung mittelbare Bindungswirkung zu verleihen:

a) Die Resolutionen der UN-Generalversammlung könnten zunächst auch als nachfolgende Staatenpraxis angesehen werden, die nach Praxis, Literatur und Rechtsprechung ${ }^{17}$ sowie nach Art. 31 III Buchst. b der Wiener Vertragsrechtskonvention bei der Auslegung der UN-Charta zu berücksichtigen ist. In der Tat wird man unter einer solchen Praxis nicht nur auf Einzelfälle bezogene Realakte, sondern auch die Abgabe genereller Erklärungen zu verstehen haben. Insoweit entstehen aber drei Probleme. Einmal kann die Praxis nicht herangezogen werden, wenn wie bei der Economic Charter einige der Vertragšpartner sich gegen diese Auslegung gewendet haben ${ }^{\mathbf{1 8}}$. Zweitens ist der Rückgriff auf die Staatenpraxis bei der Satzung einer internationalen Organisation generell fraglich, weil die Satzung die Praxis der Organe und nicht umgekehrt die Praxis der Organe die Satzungsauslegung lenken soll ${ }^{19}$. Drittens sind die einschlägigen Bestimmungen der UN-Satzung über die Förderung der wirtschaftlichen und sozialen Entwicklung und der sozialen und politischen Menschenrechte so vage ${ }^{20}$, daß man ohne Eigenverbindlichkeit der Resolution die Verbindlichkeit der

\footnotetext{
5 Vgl. derselbe, Subventionsrecht, 1978, S. 83 f.

6 Vgl. A. Verdross/B. Simma, Universelles Völkerrecht, 1976, S. 343.

7 Vgl. W. A. Kewening, Der Grundsatz der Nichtdiskriminierung im Völkerrecht der internationalen Handelsbeziehungen, 1972.

8 Vgl. G. Jaenicke, Diskriminierung, in: Strupp-Schlochauer, Wörterbuch des Völkerrechts, Bd. I, 1960, S. 387.

9 A/RES/3281 (XXIX) UNMC 1975, Nr. 1, 108 = ILM 14, 1975, 251.

$10 \mathrm{Vgl}$. C. Tomuschat, Die Charta der wirtschaftlichen Rechte und Pflichten der Staaten, ZaöRV 36 (1976), S. 444.

11 Ebenda.

12 Ebenda.

13 Ebenda.

14 Ebenda.

15 Ebenda.

16 J. A. Frowein, Der Beitrag der internationalen Organisationen zur Entwicklung des Völkerrechts, ZaöRV 36 (1976), S. 147.

17 R. Bernhardt, Die Auslegung völkerrechtlicher Verträge, 1963.

18 C. Tomuschat, a. a. O. (Anm. 10).

19 So schon für das Gewohnheitsrecht im Europäischen Gemeinschaftsrecht A. Bleckmann, Europarecht, 2. Aufl. 1978, S. 78; zur Auslegung des Gemeinschaftsrechts anhand der Praxis vgl. derselbe, Teleologie und dynamische Auslegung im Europäischen Gemeinschaftsrecht, EuR 1979.

20 C. Tomuschat, a. a. O. (Anm. 10).
} 
Konkretisierung kaum aus der UN-Satzung allein ableiten kann. Diese Bedenken bestehen auch gegen die Auffassung der Entwicklungsländer, die Economic Charter als Konkretisierung der nach der UN-Charter verbindlichen Rechtsquelle ${ }^{21}$ „Gerechtigkeit" verstehen. b) Die Resolutionen der UN-Generalversammlung können ferner über das völkerrechtliche Verbot des Rechtsmißbrauchs und über die Regeln des Ius cogens verbindlich werden ${ }^{22}$. Der Rechtsmißbrauch ist im Völkerrecht zwar ein sehr umstrittenes Rechtsinstitut ${ }^{23}$. Man wird über die bisherigen Ansätze, den Rechtsmißbrauch aus der im Völkerrecht verbindlichen materiellen Gerechtigkeit ${ }^{24}$, insbesondere aus dem Willkürverbot ${ }^{25}$, aus der Praxis der Staaten ${ }^{26}$ und aus dem Vergleich der nationalen Rechtsinstitute des abus de droit ${ }^{27}$ und des détournment de pouvoir ${ }^{28}$ abzuleiten, auf weitere, neue Gedanken zurückgreifen können und müssen, um den Rechtsmißbrauch zu stützen. Zunächst einmal ist festzuhalten, daß der Gedanke des détournment de pouvoir heute auch im Völkerrecht, nämlich in Art. 18 EMRK und im Art. 173 EWGV verankert ist und durch die modernere Auffassung, das Völkerrecht weise den Staaten zwar weite, aber eben doch begrenzte und damit durch den Gedanken des détournment de pouvoir weiter begrenzbare Kompetenzen $\mathrm{zu}^{\mathbf{2 9}}$, gefördert wird. Daneben ist zu berücksichtigen, daß nach dem Privatrecht fast aller Staaten der Verstoß gegen allgemeine Interessen und zwingende Normen Verträge vernichtet ${ }^{30}$, und daß dieser Gedanke zumindest punktuell durch die Anerkennung von ius cogens im Völkerrecht bestätigt wird. Dabei ist zu beachten, daß die betreffenden Allgemeininteressen gerade auch die Interessen der Völkerrechtsgemeinschaft umfassen ${ }^{\mathbf{3 1}}$; es ist aber anzunehmen, daß, wenn der Verstoß gegen Interessen der Völkerrechtsgemeinschaft schon nach innerstaatlichem Recht Verträge vernichtet, dies um so mehr für das Völkerrecht gilt. Die Berücksichtigung von Interessen der höheren Rechtsgemeinschaft und der Nachbarstaaten spielt auch im Bundesstaat bei der Bundestreue ${ }^{33}$ eine Rolle; es ist anzunehmen, daß diese Gedanken angesichts der wachsenden Integration der Völkerrechtsgemeinschaft und der zunehmenden Anerkennung von Allgemeininteressen der Völkerrechtsgemeinschaft ${ }^{34}$ auch in das Völkerrecht übernommen werden kann. Daß dies möglich ist, zeigt sich etwa im völkerrechtlichen Nachbarrecht ${ }^{35}$, im internationalen Wasserrecht ${ }^{36}$ und im internationalen Umweltschutzrecht - Rechtsgebiete, in denen jeder Staat die Interessen der Nachbarstaaten mitberücksichtigen muß - sowie etwa

21 Vgl. die Präambel der UN-Charta.

22 Dazu A. Bleckmann, Die Aufgaben einer Methodenlehre des Völkerrechts, 1978, S. 57 ff.

23 E. R. C. van Bogaert, Het rechtsmisbruik in het volkenrecht, 1948; H. C. Gutteridge, Abuse of Rights, Cambridge Law Journal 5 (1933), S. 22 ff.; A. C. Kiss, L'abus du droit en droit international, 1953; R. Laun, Bemerkungen zum freien Ermessen und zum detournement de pouvoir im staatlichen und im Völkerrecht, in: Festschr. f. Herbert Kraus, 1954, S. 128 ff.; H. Lauterpacht, The Function of Law in the International Community, 1933, S. 286 ff.; G. Leibholz, Das Verbot der Willkür und des Ermessensmißbrauchs im völkerrechtlichen Verkehr der Staaten, ZaöRV(1929), S. 77; G. van der Molen, Misbruik van recht in het Volkenrecht, 1949; N. Politis, Le problème des limitations de la souveraineté et la théorie de l'abus des droits dans les rapports internationaux, Recueil des cours, Bd. 6, 1925 I, S. 5 ff., 77 ff.; J. D. Roulet, Le caractère artificiel de la théorie de l'abus de droit en droit international public, 1958; M. Scerni, L'abuso di diritto nei rapporti internazionali, 1930; H. J. Schlochauer, Die Theorie des abus de droit im Völkerrecht, ZVR 17 (1933), S. 373 ff.; A. Schüle, Rechtsmißbrauch, in: Strupp/Schlochauer, Wörterbuch des Völkerrechts, Bd. 3, 1962 , S. 69 ff.; G. Schwarzenberger, Uses and Abuses of the "Abuse of Rights", Transactions of the Grotius Society 42, 1956, S. $147 \mathrm{ff}$.; Selea Trifu, La nation de l'abus du droit dans le droit international, 1940.

24 So G. Leibholz und N. Politis, a. a. O. (Anm. 23).

25 So G. Leibholz, a. a. O. (Anm. 23).

26 So A. C. Kiss, a. a. O. (Anm. 23).

27 So N. Politis, a. a. O. (Anm. 23).

28 Ebenda.

29 Dazu A. Bleckmann, Die Handlungsfreiheit der Staaten, OZöR 1978, S. 1113; derselbe, Die Aufgaben einer Methodenlehre des Völkerrechts, a. a. O. (Anm. 22), S. 33.

30 K. Simitis, Gute Sitten und ordre public, 1960.

31 Dazu A. Bleckmann, Der Grundsatz der Völkerrechtsfreundlichkeit der deutschen Rechtsordnung, DOV 1979.

32 Dazu H. W. Bayer, Die Bundestreue, 1961; J. Schmidt, Der Bundesstaat und das Verfassungsprinzip der Bundestreue, 1967; grundlegend: BVerfGE 1, 299 f., 315.

33 A. Bleckmann, Art. 5 EWG-Vertrag und die Gemeinschaftstreue, DVBl. 1976, S. 483.

34 A. Bleckmann, Die Aufgaben einer Methodenlehre des Völkerrechts, a. a. O. (Anrn. 22), S. $11 \mathrm{ff}$.

35 Dazu G. Ress, Die Rechtslage Deutschlands nach dem Grundlagenvertrag vom 21. Dezember 1972, S. 233 ff. m.w.N

36 Skeptisch: F. Berber, Die Rechtsquellen des intertnationalen Wassernutzungsrechts, 1955; vgl. aber die Helsinki-Rules der ILA. 
in Art. 24 der Economic Charter, wonach "All States have the duty to conduct their mutual economic relations in a manner which takes into account the interest of other countries. In particular, all States should avoid prejudicing the interestes of developing countries", und in Art. 103 I 1 EWGV, nach dem ,Die Mitgliedsstaaten . . . ihre Konjunkturpolitik als eine Angelegenheit von gemeinsamen Interesse" betrachten. Auch das im Repressalienrecht ${ }^{37}$ sowie bei sonstigen Eingriffen in Rechte fremder Staaten ${ }^{38}$ anwendbare, aus dem nationalen Rechtskreis übernommene ${ }^{39}$ und auch in dern EMRK ${ }^{40}$ und bei den Grundrechten der Europäischen Gemeinschaft ${ }^{\mathbf{4 1}}$ anwendbare Verhältnismäßigkeitsprinzip verlangt eine Abwägung der beteiligten Interessen. Auch die im deutschen Verfassungsrecht hinsichtlich der Beschränkbarkeit von Grundrechten entwickelte Mißbrauchstheorie ${ }^{\mathbf{4 2}}$, nach der alle Grundrechte im Zusammenspiel der verschiedenen privaten und öffentlichen Interessen notwendig immanente Grenze haben, muß hier herangezogen werden. Und schließlich ist generell auf die Gerechtigkeitsvorstellung der Interessenjurisprudenz $z^{43}$ zu verweisen, die auch im Völkerrecht eine Heimstatt hat ${ }^{44}$ und die Abwägung zwischen den beteiligten Interessen verlangt.

Damit kommen wir zu dem Ergebnis, daß das Verbot des Rechtsmißbrauchs auch im Völkerrecht eine hinreichende Grundlage findet und den Staaten verbietet, gegen überwiegende Interessen der Allgemeinheit und fremder Staaten verstoßen. Die Interessen der Allgemeinheit können nun in bindenden internationalen Verträgen, etwa in den Präambeln der Satzungen der UNO und ihrer Sonderorganisationen, aber auch in unverbindlichen internationalen Akten wie gerade den Resolutionen der UN-Vollversammlung verankert sein. Denn die Eigenart des Rechtsmißbrauchs ist ja gerade, den Verstoß auch gegen noch nicht bindende Allgemeininteressen der Völkerrechtsgemeinschaft zu verbieten.

c) In Präzisierung einer allgemeinen Theorie von Verdross ${ }^{\mathbf{4 5}}$ haben Verdross/Simma ${ }^{\mathbf{4 6}}$ nunmehr die Satzung der UNO als Verfassung der Völkerrechtsgemeinschaft verstanden. Diese Sicht muß langfristig zu einer Revolutionierung des Völkerrechts führen. Sie hat zahlreiche Konsequenzen, die hier nicht abschließend entwickelt werden können ${ }^{47}$. Eine wichtige, von Verdross/Simma selbst gezogene Konsequen $z^{48}$ ist, daß sich - wie das gesamte nationale Recht von der nationalen Verfassung - das gesamte Völkerrecht von der UN-Satzung ableiten lassen muß. Das scheint insoweit keine Veränderung zu bewirken, als die UN-Satzung und das übrige UN-Recht, insbesondere Art. 38 des IGH-Statuts, die bisherigen Rechtsquellen und den bisherigen Inhalt des Völkerrechts anerkennen. Eine weitere Folge aber ist, daß das gesamte Völkerrecht durch die Brille des UN-Rechts gesehen werden müßte. Konkretisierungen des UN-Rechts durch Resolutionen der UN-Vollversammlung wirken sich nach einer solchen Konzeption unmittelbar im allgemeinen Völkerrecht aus; den UN-Resolutionen käme bei dieser Sicht sogar ein besonders hoher, wenn nicht der höchste Stellenwert bei der Feststellung, Auslegung und Konkretisierung des Völkerrechts zu.

\footnotetext{
37 Vgl. A. Bleckmann, Zwangsmittel im Gemeinsamen Markt. Die Repressalie im Europäischen Gemeinschaftsrecht, RIW/AWD 1978, S. 91 m.w.N.

38 H. Mosler, Völkerrecht als Rechtsordnung, ZaöRV 36 (1976), S. 6, 45.

39 Vgl. BVerfGE 16, 194, 201 f.; R. von Kraus, Der Grundsatz der Verhältnismäßigkeit, 1955; M. Gentz, Zur Verhältnismäßigkeit von Grundrechtseingriffen, NJW 1968, S. 1600 mit zahlreichen Nachw. der Rspr. des BVerfG.

$40 \mathrm{Vgl}$. H. Mosler, a. a. O. (Anm. 38).

41 A. Bleckmann, Europarecht, a. a. O. (Anm. 19), S. 84.

42 Dazu H. U. Gallwas, Der Mißbrauch von Grundrechten, 1967, S. 17 ff.; vgl. auch BVerfGE 7, 198, 210; 12, 1, 4.

43 Dazu G. Ellscheid/W. Hassemer (Hersg.), Interessenjurisprudenz, 1974.

44 Vgl. A. Bleckmann, Die Aufgaben einer Methodenlehre des Völkerrechts, a. a. O. (Anm. 22), S. 10 ff. m. w. N.

45 A. Verdross, Die Verfassung der Völkerrechtsgemeinschaft, 1926.

46 A. Verdross/B. Simma, a. a. O. (Anm. 6), S. $71 \mathrm{ff}$.

47 Dazu A. Bleckmann, Die Aufgaben einer Methodenlehre des Völkerrechts, a. a. O. (Anm. 22).

48 A. Verdross/B. Simma, a. a. O. (Anm. 6), S. 200.
} 
d) Nach den oben zitierten Ausführungen von Tomuschat und Frowein sind Resolutionen der UNO zwar nicht absolut verbindlich, schränken aber den rechtlichen Argumentationsrahmen ein.

(1) Das bisherige ,,Kästchendenken“ in den drei in Art. 38 IGH-Statut genannten Rechtsquellen ist mit solchen Gedanken unvereinbar. Nach dieser traditionellen Auffassung beruhen das Völkervertragsrecht auf dem Willen der Staaten, das Völkergewohnheitsrecht auf der Praxis der Staaten und die allgemeinen Rechtsgrundsätze auf dem Vergleich des nationalen Rechts. Resolutionen der Generalversammlung - wenn sie nicht als Vertrag, als Staatenpraxis oder als allgemeine Rechtsgrundsätze verstanden werden können, wie Tomuschat und Frowein annehmen -, die in Art. 38 IGH-Statut erwähnte Lehre und die damit indirekt anerkannten wissenschaftlichen Methoden der Lehre - Vernunftsschlüsse, logische Schlüsse, Analogien usw. - haben in diesem System offensichtlich keinen Platz. Uberdies läuft das „Kästchendenken“ so ab, daß eine bestimmte Lösung zunächst auf Vertragsrecht, dann auf Gewohnheitsrecht, schließlich auf allgemeine Rechtsgrundsätze zu stützen versucht wird. Ist die Praxis unzureichend oder fehlt die notwendige Rechtsüberzeugung, ist Gewohnheitsrecht zu verneinen. Findet sich ein Rechtssatz nur in wenigen Rechtsordnungen, fehlt ein allgemeiner Rechtsgrundsatz. Eine Sicht, die bei der Uberzeugungsbildung des Richters den Rechtssatz gleichzeitig auf ,, wenig" Staatenpraxis, ,, wenig" U'bereinstimmung der nationalen Lösung, ein ,,bißchen“ Analogie usw. stützt, selbst wenn Gewohnheitsrecht und allgemeine Rechtsgrundsätze an sich abzulehnen wären, ist nach dieser Konzeption nicht möglich.

Einen gewissen Ausweg aus dieser Situation sieht die Lehre in der Anerkennung weiterer Rechtsquellen. Die Aufzählung der Rechtsquellen in Art. 38 IGH-Statut soll nicht abschließend sein; es gebe keinen Numerus clausus der völkerrechtlichen Rechtsquellen ${ }^{49}$. Der Nachteil dieser Konzeption ist, daß den Resolutionen der UN-Vollversammlung, der Lehre und ihren Methoden dann ein zu starkes Gewicht beigemessen wird. Der Rechtssatz kann nach dieser Konzeption allein auf Resolutionen der Generalversammlung, auf den consensus doctorum, auf die Analogie usw. gestützt werden. Richtiger ist es wohl, nur die drei Rechtsquellen Vertragsrecht, Gewohnheitsrecht und allgemeine Rechtsgrundsätze anzuerkennen, diese Rechtssätze aber in der Sicht der UN-Resolutionen und der Lehre festzustellen und zu konkretisieren.

Noch besser erscheint mir, mit der modernen Lehre ${ }^{50}$ auf das Kästchendenken in verschiedenen Rechtsquellen zu verzichten und auf die faktische Úberzeugungsbildung der Staaten oder eines Richters abzustellen. Den ersten Schritt zu einer solchen Auffassung hat Simma getan $^{51}$, nach dem die Rechtsquellen des Art. 38 IGH-Statut nur Ausdruck einer einzigen Rechtsquelle, der Rechtsüberzeugung der Staaten sind. Die „Rechtsquellen“ des Art. 38 IHG-Statut bestimmen dann nur das Verfahren, in dem diese Rechtsüberzeugung festgestellt werden kann. Dabei sind die Verfahrensarten des Art. 38 IHG-Statut nicht abschlieBend ${ }^{52}$. Kommt es aber nur auf die Feststellung der Rechtsüberzeugung der Staaten an, kann diese durch die gleichzeitige Heranziehung von Praxis, Rechtsvergleichung und Resolutionen der Generalversammlung bewiesen werden. Daß die Praxis für Völkergewohnheitsrecht an sich nicht ausreicht, daß der Rechtssatz sich in so wenigen Rechtsordnungen findet, daß ein allgemeiner Rechtsgrundsatz zu verneinen ist, schließt dann nicht aus, daß die Rechts-

\footnotetext{
49 Vgl. C. Tomuschat, a. a. O. (Anm. 10).

50 Vgl. J. P. Müller, a. a. O. (Anm. 2).

51 B. Simma, Methodik und Bedeutung der Arbeit der UN für die Fortentwicklung des Völkerrechts, in: W. A. Kewenig (Hrsg.), Die UN im Wandel, 1975, S. 79 ff.; A. Verdross/B. Simma, a. a. O. (Anm. 6), S. 358 ff.; dazu C. Tomuschat, a. a. O. (Anm. 10); A. Bleckmann, Die Aufgaben einer Methodenlehre des Völkerrechts, a. a. O. (Anm. 22), S. 29.

52 Vgl. dazu C. Tomuschat, a. a. O. (Anm. 10).
} 
überzeugung der Staaten durch den gleichzeitigen Rückgriff auf dieses Material bewiesen wird, daß die gleichzeitige Sicht der Praxis, der nationalen Rechtssätze und der UN-Resolutionen die Feststellung der Rechtsüberzeugung der Staaten gestattet.

Allerdings hat diese Sicht immer noch den Nachteil, daß man die Auffassung der Lehre und ihre Methoden, etwa die Analogie, nicht berücksichtigen kann. Das ist nur möglich, wenn man darauf abstellt, daß die rechtliche Argumentation den Partner, eventuell das Gericht überzeugen muß, und daß das internationale Gericht sich selbst von einer bestimmten Lösung überzeugen muß. Bei dieser Uberzeugungsbildung aber kann nun das gesamte Material der Staatenpraxis, der Rechtsvergleichung, der UN-Resolutionen, der Lehre, der Methoden der Lehre usw. gleichzeitig berücksichtigt werden. Mir scheint, daß die Lehre und Rechtsprechung einem solchen Verfahren weithin tatsächlich schon immer folgen.

(2) Diese neue Lehre hebt zu Recht hervor, daß das Völkerrecht nichts Objektiv-Statisches, sondern ein dynamisches Verfahren ist. Es kommt nicht so sehr darauf an, ein bestimmtes ,,einzig richtiges“ Ergebnis zu finden, weil die Gerichtsbarkeit im internationalen Raum sehr selten ist. Entscheidend ist vielmehr, daß das Völkerrecht den Argumentationshaushalt der Streitparteien auf bestimmte rechtliche Inhalte und Methoden beschränkt, so die Verhandlungen versachlicht und die Konzeption einander annähert, was zu einem besseren Kompromiß führen kann.

3. Zu dem von Weber zitierten Gedanken Ingo v. Münchs ${ }^{53}$, die Úbertragbarkeit des nationalen Sozialstaatsprinzips, das Leistungsansprüche der Individuen gegen den Staat auf Sozialhilfe begründet ${ }^{54}$, in das Völkerrecht zu prüfen, ist hier wenig nachzutragen. Diese Ubertragung scheitert sicherlich nicht schon daran, daß nur wenige Staaten - soweit ersichtlich nur die Bundesrepublik und die V. Französische Republik - das Sozialstaatsprinzip ausdrücklich in die Verfassung übernommen haben. Ähnlich wie das Rechtsstaatsprinzip die gesamte Rechtsordnung der meisten Staaten prägt, steht hinter der modernen Sozialstaatsgesetzgebung zumindest stillschweigend das Sozialstaatsprinzip. Die Úbertragung soll vielmehr nach von Münch daran scheitern, daß die Völkerrechtsordnung noch zu wenig solidarisch ist. Die Strukturen der Völkerrechtsordnung verbieten also die Úbernahme des Sozialstaatsprinzips in das Völkerrecht ${ }^{\mathbf{5 5}}$, während das Rechtsstaatsprinzip ${ }^{\mathbf{6 6}}$ und das Sozialstaatsprinzip ${ }^{57}$ in das Europäische Gemeinschaftsrecht übernommen werden könnten. Ob diese Auffassung ganz richtig ist, kann sich nur aus einer genauen Untersuchung der Struktur der Völkerrechtsordnung ergeben. Dabei spielen insbesondere die verschiedenen hier untersuchten Gründe für einen Anspruch auf Entwicklungshilfe eine Rolle. Sprechen diese verschiedenen Gründe für einen solchen Anspruch, ergibt sich, daß die Völkerrechtsgemeinschaft hinreichend solidarisch ist, das Sozialstaatsprinzip zu tragen. Dann kann das Sozialstaatsprinzip als weitere Verstärkung solcher Ansprüche herangezogen werden. Dabei ist vor allem auch zu beachten, daß das Sozialstaatsprinzip in der universellen Sozialcharta verankert sein kann. Ob auf die Sozialcharta auch Ansprüche dritter Staaten gestützt werden können, werden wir später untersuchen.

4. Auch zu den Ausführungen Webers zur Konstruktion eines ,Folgenbeseitigungsanspruchs" durch A. Leitolf58 ist einiges nachzutragen. Es kann sich dabei sowohl um völkerrechtliche Schadensersatzansprüche als auch um Bereicherungsansprüche handeln, die ent-

\footnotetext{
53 Ingo von Münch, Die Zeit, No. 8 v. 11. 2. 1977, S. 54.

$54 \mathrm{Vgl}$. A. Bleckmann, Subventionsrecht, a. a. O. (Anm. 5), S. 32.

55 Vgl. derselbe, Zur Strukturanalyse im Völkerrrecht, Rechtstheorie 1978, S. 143.

56 Derselbe, Der Rechtsstaat in vergleichender Sicht. Zugleich ein Beitrag zur Rechtsquellenlehre des Europäischen Gemeinschaftsrechts, Jahrb. f. internationales Recht 20 (1977), S. 406.

57 Derselbe, Die Rechtsvergleichung im Europäischen Gemeinschaftsvergleich im Bezugsrahmen internationalen und supranationalen Rechts, 1978, S. 97.

58 A. Leitolf, Blätter für deutsche und internationale Politik, 1976, S. 166.
} 
weder mit der Kolonisierung oder aber mit der Ungerechtigkeit der heutigen Weltwirtschaftsordnung begründet werden könnten:

a) Schwierig ist die Begründung, wenn man auf die vergangene Kolonisierung abstellt:

(1) Nach den Prinzipien des völkerrechtlichen Schadensersatzrechts müßten die Begründung und die wirtschaftliche Ausbeutung der Kolonien rechtswidrig gewesen sein. Dabei kommt es nach den Regeln des völkerrechtlichen intertemporellen Kollisionsrechts auf den Verstoß gegen das damals geltende Völkerrecht an. Nach dem damaligen Recht waren die Kolonisierung und die Ausbeutung der Kolonien aber sicherlich nicht völkerrechtswidrig. Es kann dahingestellt bleiben, ob in der neueren Konzeption die Kolonisierung und die Ausbeutung von Kolonien rechtswidrig ist oder ob nur eine Verpflichtung besteht, die Staaten in die Unabhängigkeit zu entlassen. Denn die dahingehende Auffassung der Neustaaten kann nicht als Naturrecht verstanden werden, das allein rückwirkend gelten könnte. Dem widerspräche die positivrechtliche Ausrichtung des Völkerrechts.

(2) Eine eventuelle Bereicherung der Mutterstaaten und ihrer Angehörigen fände dagegen eine Rechtsgrundlage im damaligen Völker- und nationalen Recht. Insoweit kann man von einer ungerechtfertigten Bereicherung nicht ausgehen. Allerdings wird die nach Völkerrecht erforderliche rechtliche Entschädigung bei der Enteignung fremder Gesellschaften, welche ihre Konzession während der Kolonialzeit erlangt haben, durch die Neustaaten häufig mit der Begründung abgelehnt, diese Gesellschaften hätten sich während der Kolonialzeit ungerechtfertigt bereichert, so daß die Bereicherung von der Entschädigung abzuziehen sei ${ }^{59}$. Ob diese Rechtsauffassung richtig ist, kann hier dahingestellt bleiben. Einen völkerrechtlichen Bereicherungsanspruch der ehemaligen Kolonien gegen ihre Mutterstaaten vermag sie wohl nicht zu tragen.

(3) Drittens ist fraglich, ob gerade die Mutterstaaten diesen Schaden zugefügt und sich bereichert haben. Sehe ich die Kolonialzeit richtig, haben die Mutterstaaten meist unmittelbar Gewinn aus den Kolonien nicht gezogen, sondern in der Regel noch Zuschüsse zum Haushalt der Kolonien geleistet. Es ist aber fraglich, ob die Mutterstaaten sich die Gewinne ihrer Angehörigen und Gesellschaften zurechnen lassen müssen, und ob auf der anderen Seite die nur mittelbare Bereicherung der ganzen Bevölkerung des Mutterlandes und damit des Staates selbst (gut bezahlte Arbeitsmöglichkeiten für Staatsangehörige in den Kolonien; Märkte für die Industrieproduktion des Mutterlandes; billige Rohstoffe für die Industrie des Mutterlandes: insgesamt gesehen, ein Beitrag zum Wirtschaftswachstum, zur Vollbeschäftigung, zur Erhöhung des Lebensstandards, zum Zahlungsbilanzausgleich des Mutterlandes usw.) für eine rechtliche Bereicherung des Mutterlandes ausreicht. Auf der anderen Seite hat die Kolonisierung, das muß einmal deutlich gesagt werden, den Kolonien eben nicht nur geschadet, sondern ihnen auch Vorteile gebracht, die bei einer solchen Abrechnung mitberücksichtigt werden müßten. Es ist eben nicht so, daß die hypothetische Entwicklung der Neustaaten ohne Kolonisierung ins goldene Zeitalter geführt hätte. Hier ist die friedensstiftende Funktion der Kolonialverwaltung einzuordnen, die Tatsache, daß die Ubernahme der Sprache und des Rechts des Mutterlandes erst funktionierende Gemeinwesen geschaffen und die notwendige nationale Einheit begründet haben. Auch der Beitrag der Mutterländer zur wirtschaftlichen und sozialen Entwicklung der Kolonien sollte schließlich nicht so niedrig veranschlagt werden, wie das heute weithin üblich ist. Ich verkenne dabei nicht, daß die Kolonisierung meist auch viel Leid für die ansässige Bevölkerung mit sich brachte.

(4) Solche Wiedergutmachungs- und Bereicherungsansprüche bestünden ferner höchstens im Verhältnis der ehemaligen Mutterländer zu ihren Kolonien. Der gesamten westlichen

59 Reichert-Facilides, Internationalrechtliche Betrachtungen zur Dekolonisierung, 1964. 
Welt die , Schuld“ für das Kolonialregime zuzuordnen, weil sie es rechtlich mitgetragen hat, die ganze westliche Welt als bereichert anzusehen, weil die Kolonien indirekt den Reichtum der ganzen westlichen Welt gefördert haben, würde die Zurechnungsmöglichkeiten des völkerrechtlichen Schadens- und Bereicherungsrechts wohl überspannen.

(5) $\mathrm{Zu}$ berücksichtigen ist ferner juristisch auch, daß die Kolonien noch keine Völkerrechtssubjekte waren. Den Schadens- und Bereicherungsanspruch auf einen späteren Zeitpunkt, nämlich auf die Zeit der Bildung der Neustaaten zu verlegen, erfordert schwierigste juristische Konstruktionen.

(6) Wir haben aus dem Versailler Vertrag gelernt, welche Emotionen die Festschreibung von Reparationsforderungen nach sich zieht. Die oben skizzierte „Abrechnung“ $z$ wischen den Mutterländern und den Neustaaten würde die internationalen Beziehungen zwischen Nord und Süd einer noch schwereren Belastungsprobe unterziehen, als dies heute schon der Fall ist. Auch diese rechtspolitischen Gründe sollte man bei der rechtlichen Konstruktion mit berücksichtigen.

b) Man könnte Schadensersatz- und Bereicherungsansprüche ferner auf die Tatsache stützen, daß die heutigen Wirtschaftsstrukturen ungerecht sind, die entwickelten Länder sich also aufgrund dieser Strukturen zu Lasten der Entwicklungsstaaten ständig ungerechtfertigt bereichern. Das ist sicherlich bis zu einem gewissen Maße richtig und trägt einen Bereicherungsanspruch dem Grund nach ${ }^{80}$. Allerdings würde eine genaue Berechnung der Anspruchshöhe voraussetzen, daß man die Ergebnisse einer gerechten Wirtschaftsordnung mit den heutigen Ergebnissen der Weltwirtschaftsordnung vergleicht. Gerade für eine gerechte Wirtschaftsordnung haben wir heute aber noch keine Maßstäbe. Wie Tomuschat nachgewiesen hat, kann die Economic Charter nicht als ein gerechter Interessenausgleich verstanden werden, weil sie umgekehrt die Interessen der Entwicklungsstaaten zu Lasten der Indusrienationen einseitig zu stark betont ${ }^{\mathbf{6 1}}$. Uberdies scheint eine Prognose der wirtschaftlichen Ergebnisse einer gerechteren internationalen Wirtschaftsordnung unmöglich zu sein.

5. Inamul $\mathrm{Haq}^{62}$ versucht, den Anspruch auf Entwicklungshilfe auf die internationalen Menschenrechte zu stützen. Auch die Economic Charter scheint diesen Weg zu gehen, weil sie als Rechtsgrundlagen der Charter auch die internationalen Menschenrechte heranzieht. Dabei muß man davon ausgehen, daß die Bestimmungen der UN-Charta über die Menschenrechte und die Pflichten zur Mitarbeit bei der wirtschaftlichen und sozialen Entwicklung der gesamten Welt zu vage sind, um Pflichten zu begründen, und daß die allgemeine Menschenrechtsdeklaration von 1948 die Staaten nicht bindet. Rückgreifen kann man damit nur auf die universellen Pakte von 1964 und auf bestimmte Konventionen der ILO. Insbesondere der Covenant on Economic, Social and Cultural Rights anerkennt u. a. ein Recht auf Arbeit, ein Recht auf gerechte Arbeitsbedingungen, insbesondere auf gerechte Entlohnung, das Recht auf angemessene Ernährung, Kleidung und Wohnung, das Recht auf angemessenen Lebensstandard und dessen ständige Verbesserung, das Recht auf Gesundheit, das Recht

60 Allerdings ist hier im Völkerrecht vieles unklar. Sicher ist nur, daß der Bereicherungsgrundsatz als allgemeiner Rechtsgrundsatz im Völkerrecht gilt (G. Dahm, Völkerrecht, Bd. II, 1962, S. 274 f.). Sicher ist auch, daß etwa die im $₫ 812$ BGB verankerten Fälle der ungerechtfertigten Bereicherung durch diesen Rechtsgrundsatz gedeckt werden. So liegt der Fall hier aber nicht, weil die einzelnen Rechtsgeschäfte im Rahmen der heutigen Wirtschaftsordnung ihren Rechtsgrund finden. Meines Erachtens muß man dem Gerechtigkeitsgedanken, der auch in der UN-Charta verankert ist, im Völkerrecht aber zumindest insoweit eine Heimstatt geben, als Vorteile, die aufgrund evident ungerechter Völkerrechtsregeln erreicht wurden, zurückgegeben sind. Dabei ist zu beachten, daß hier das oben dargelegte Problem der nur mittelbaren Bereicherung der Industriestaaten auftritt. Ferner ist zu berücksichtigen, daß in dèr neueren Zeit die Weltwirtschaftsordnung überdies durch Gewährung von Präferenzzöllen und ähnliche Maßnahmenzugunsten der Entwicklungsstaaten weitgehend an einen gerechten Standard angehoben wurde, der von den Entwicklungsstaaten gefordert wird. Der Anspruch bestünde dann nur für die Vergangenheit.

61 C. Tomuschat, a. a. O. (Anm. 10).

62 Inamul Haq, From Charity to Obligation. Legal Perspective on Commercial Resource-Transfers, Master-Thesis der Harvard-University, 1978. 
auf Bildung, das Recht auf Teilhabe am wissenschaftlichen Fortschritt. Auch die als Abwehrrechte formulierten Grundfreiheiten des Covenant on Civil and Political Rights können analog der deutschen Lehre ${ }^{63}$ u. U. unter Rückgriff auf das dem Sozialpakt zugrunde liegende Sozialstaatsprinzip mit der Begründung in Teilhaberechte umgedeutet werden, daß der Staat auch die materiellen und organisatorischen Voraussetzungen der Abwehrrechte schaffen muß. Allerdings ist dieser Rückgriff auf die deutsche Lehre recht spekulativ, weil andere Staaten die allgemeinen Grundrechtslehren ${ }^{64}$ der Bundesrepublik zum größten Teil nicht kennen und diese Lehren bisher auch nur äußerst zögernd zur Auslegung der EMRK herangezogen wurden. Dieser Vorbehalt ist auch bei der folgenden Heranziehung der deutschen Grundrechtslehren in anderen Bereichen zu beachten.

a) Von großer Bedeutung ist zunächst einmal, daß die Rechte der universellen Sozialcharta so vage formuliert sind, daß es sich nicht um echte Rechte handeln kann. Dagegen sprechen auch die übrigen Formulierungen des Sozialpakts.

b) Entscheidender noch ist, daß die Sozialcharta und die universelle Menschenrechtskonvention der Konstruktion nach ${ }^{65}$ nur Rechte des etwa auf dem Boden der Bundesrepublik lebenden Individuums A gegen die Bundesrepublik, nicht aber Rechte des etwa an der Elfenbeinküste lebenden $B$ gegen die Bundesrepublik und noch weniger Rechte der Elfenbeinküste selbst gegen die Bundesrepublik begründet. Diese enge Begrenzung der Rechte könnte man auf zwei Wegen umgehen, die allerdings heute noch recht spekulativ sind:

(1) Zunächst könnte man annehmen, daß die universellen Menschenrechtspakte, da es sich nur um Konkretisierungen der einschlägigen Bestimmungen der UN-Charta handelt, grundlegende ,Verfassungswerte“ der Völkerrechtsgemeinschaft festlegen, die über den engen Wortlaut der Pakte hinaus auf das ganze Völkerrecht ausstrahlen müssen. In der Tat wird man so annehmen müssen, daß die Grundwerte des Bürgerrechtspaktes die Vökerrechtskonzeption der Staaten auch im Verhältnis der Staaten zueinander prägen muß, soweit es sich um die Behandlung von Individuen handelt ${ }^{66}$. Darüber hinaus hat die deutsche Konzeption der Grundrechte als Werte ja gerade die Konsequenz, daß die im Wortlaut der Grundrechte verankerte Beschränkung der Bindungswirkung aufgehoben wird und die Grundrechte in allen Rechtsbeziehungen Rechtswirkungen äußern können ${ }^{67}$.

(2) Zweitens muß man das Zusammenspiel zwischen den Vorschriften der UN-Charta, welche Menschenrechte und die Pflicht zur Förderung der wirtschaftlichen und sozialen Bedingungen verankern, mit ihren Konkretisierungen, den Menschenrechtspakten beachten. Dabei ist davon auszugehen, daß die UN-Charta diese Pflichten nicht nur im Verhältnis des Staates zu seinen Gewaltunterworfenen, sondern in allen Beziehungen, also auch etwa im Verhältnis der Staaten untereinander fördern will. Dieses Wechselspiel zwischen UNCharta und Menschenrechtspakten kann in einer doppelten Sicht gesehen werden:

aa) Einmal könnten die Pakte als Konkretisierung der UN-Charta begriffen werden. In erster Linie ist dann die UN-Charta verbindlich. Durch diese ,Eingliederung "der Pakte in die UN-Satzung werden zwei Folgen ausgelöst: Einmal erstreckt sich der Kreis der Verpflichteten auf alle UN-Mitglieder, selbst wenn diese die Menschenrechtspakte nicht ratifiziert haben. Auf der anderen Seite werden die Werte der Pakte auf die Völkerrechtsbeziehungen

\footnotetext{
63 A. Bleckmann, Subventionsrecht, a. a. O. (Anm. 5), S. 33.

64 Derselbe, Grundrechte, erscheint 1979.

65 Der Wortlaut des universellen Sozialpakts enthält entgegen dem Wortlaut des universellen Bürgerrechtspakts eine Beschränkung auf Rechte der Bewohner eines Staates gegen den Gebietsstaat nicht. Er könnte deshalb in dem Sinne ausgelegt werden, daß auch der Staat A auch die Angehörigen des Entwicklungsstaates B zu fördern hat, wenn nicht die Territorialklauseln(Art. $28 \mathrm{f}$. IPwirtR, Sartorius II, Loseblattsammlung. Stand März 1978, 21) in eine andere Richtung weisen.

66 A. Bleckmann, Die Aufgaben einer Methodenlehre des Völkerrechts, a. a. O. (Anm. 22), S. 35 ff.

67 Vgl. BVerfGE 7, 198, 204 f.; ständige Rspr. etwa noch BVerfGE 35, 79, 114 f. m. w. N.
} 
zwischen den Staaten erstreckt. Dieser Weg erscheint mir ungangbar, weil die UN-Charta zu vage ist, um bestimmte Verpflichtungen zu begründen.

bb) Umgekehrt kann man die UN-Charta zur Auslegung der Pakte heranziehen. Diese Auslegungsrichtung führt zur Bestätigung der Pakte als universelle Werte, die in allen Beziehungen wirken können.

cc) In dieser Sicht fließt aus den Werten des Sozialpakts die Geltung des Sozialstaatsprinzips zwischen den Staaten. Zur Verstärkung dieses Prinzips kann auf die Rechtsvergleichung zurückgegriffen werden, wie wir dies oben angedeutet haben.

Auch die Bedenken, die im deutschen Recht gegen die Grundrechtsträgerschaft öffentlichrechtlicher Körperschaften gehegt werden ${ }^{68}$, greifen im Völkerrecht nicht. Denn im nationalen Recht wird dieser Anspruch untergeordneter Körperschaften und Organe im wesentlichen deshalb abgelehnt, weil die Stützung auf Grundrechte die Einheit der Staatsgewalt aufheben, zu einer Versteinerung der Zuständigkeit führen müßte, deren flexible Veränderung in der Hand des Staates liegen muß. Dieser Gedanke greift bei den einander koordinierten Staaten auf Völkerrechtsebene nicht. Hier ist zu bedenken, daß durch einen Anspruch auf Entwicklungshilfe im Ergebnis nur Individualansprüche auf Sozialhilfe der durch den Staat mediatisierten Individuen zusammengefaßt werden. Wie beim diplomatischen Anspruch, dem der Entwicklungshilfeanspruch dann ähnelt, tritt der Staat als Repräsentant dieser Individuen auf.

6. Aus den bisher dargestellten verschiedenen Erwägungen heraus möchte ich annehmen, daß heute vielleicht noch keine Pflicht der Industriestaaten zur Leistung von Entwicklungshilfe besteht, daß die Völkerrechtsordnung sich aber in dieser Richtung entwickelt und heute schon sogenanntes ,,soft law" besteht. Allerdings müssen wir uns über diese Pflicht noch nähere Klarheit verschaffen:

a) Ebensowenig wie bei den Teilhaberechten der deutschen Grundrechtslehre kann aus den oben entwickelten Grundlagen für eine Pflicht zur Leistung von Entwicklungshilfe eine auch nur angenäherte Angabe über die Art und Höhe der Entwicklungshilfe abgeleitet werden. Man kann insoweit höchstens eine Pflicht zur Leistung einer ,,angemessenen“ Entwicklungshilfe annehmen, bei der die Interessen der Entwicklungsstaaten mit den Interessen der Industrienationen abgewogen werden müssen. Ein Industriestaat, der überhaupt keine Entwicklungshilfe leistet, würde aber wohl heute schon gegen das Völkerrecht verstoßen. b) Noch schwieriger ist der Kreis der Anspruchsberechtigten und der Anspruchsverpflichteten zu bestimmen. Sicherlich ist es aufgrund der Kriterien und Listen der internationalen Organisationen möglich, den Kreis der Entwicklungsstaaten und der Industrienationen festzulegen. Eine Konkretisierung der Pflicht des Staates A, gerade dem Staat B eine bestimmte Form der Entwicklungshilfe in bestimmter Höhe zu leisten, ist aber nicht möglich. Man kann auch nicht davon ausgehen, daß es sich bei der Entwicklungshilfe um einen Kollektivanspruch (Gesamtgläubigerschaft, Gesamthand?) gegen alle Industriestaaten (Gesamtverpflichtung, Gesamtschuld?) handelt. Folglich ist ein Anspruch eines bestimmten Staates oder aber aller Entwicklungsstaaten zusammen gegen einen bestimmten Industriestaat oder gegen die Gesamtheit der Industriestaaten auszuschließen. Es besteht nur eine objektive Pflicht der Industriestaaten gegenüber der Staatengemeinschaft, Entwicklungshilfe zu leisten. Fraglich ist dabei, ob die UNO in diesem Sinne Repräsentant der Staatengemeinschaft ist und von einem bestimmten Staat im Namen der Völkerrechtsgemeinschaft eine Befolgung dieser objektiven Pflicht verlangen kann. 


\title{
A Claim to Foreign Aid?
}

\author{
By Albert BleckmanN
}

The article discusses potential bases for claims of developing countries to foreign aid under international law. The current aid-practice cannot by itself create such a claim, but it is not completely irrelevant. It might become relevant under the principle of estoppel. The Charter of Economic Rights and Duties of States of 1974 only has the character of "soft law". As such, it might be a factor in establishing "abus de droit". Within this framework, the article submits that resolutions of the UN, doctrine, practice, comparative law etc. as a whole might be used to form the legal opinion of an international judge. Therefore it does not matter that state practice or comparative law alone cannot constitute a rule of law. In addition the article discusses whether the "Sozialstaatsprinzip" can be found in international law and whether a claim to foreign aid can be derived from the injustices of colonialism or the present economic order or from international human rights. As a result, the author states that while today there is no claim under international law to a specific amount of foreign aid, legal development points in this direction, and the refusal of any foreign aid at all is already a violation of international law.

\section{The Common Fund}

\section{BY JOACHIM BETZ}

The political attitude of the major industrial countries towards the demands of the Group of 77 for a Common Fund changed considerably in course of time. Critisizing the Fund initially as an instrument for the bureaucratic reshaping of the world commodity trade, these countries accepted at last the idea of price stabilization for raw materials and put forward on their part proposals for the setting up of a Common Fund.

This Fund has in fact never been, according to its conception, an attempt at global planning but an attempt at stabilizing commodity prices along the long-term market trend and to improve the bargaining position of the commodity exporting developing countries. On the other hand, the Common Fund (as proposed by the Group of 77) is not an instrument totally devoid of aid elements as it contains some scarcely disguised subsidies for the financing of stocking activities and other measures. The readiness of the industrial countries to accept finally the financing of price stabilization measures on a much broader scale than they were prepared to hitherto, is due to their changed perceptions of the possible merits and dangers of a Common Fund and, above all, to the fact that they are inable to make concessions in other important fields (trade, technology and resource transfer) of the North-South dialogue. It will be important in the course of the ongoing negotiations on the Common Fund and on new commodity aggreements - especially in consideration of the already considerably diverging interests of the producing countries themselves - to optimize and not to mimimize their results. Otherwise we will see the establishment of just another impotent mini-fund which will hardly be able to tackle the global commodity problems. 